\title{
Narrow stripe AIGaAs lasers using double current confinement
}

\author{
H. Blauvelt, S. Margalit, and A. Yariv \\ California Institute of Technology, Pasadena, California 91125
}

(Received 6 July 1982; accepted for publication 24 August 1982)

Gain guided AlGaAs lasers in which the current is restricted to flow between two narrow stripes have been fabricated. The double current confinement configuration, which is fabricated by a selective meltback-growth technique, enables the current injection to be restricted to a very narrow section of the active layer. These lasers exhibit very strong antiguiding and operate in many longitudinal modes, which are characteristics of narrow stripe lasers. Potential applications of the twin vertical stripe configuration include arrays of optically coupled lasers and, if a real index waveguiding mechanism can be combined with double current confinement, low threshold lasers.

PACS numbers: $42.55 . P x, 42.80 . S a$

One of the most widely studied classes of injection lasers are the gain guided stripe geometry lasers. The primary advantage of these laser structures is the simplicity of their fabrication. However, there are several disadvantages of gain guided stripe lasers, such as higher threshold currents than the more complicated buried heterostructure and transverse junction stripe laser structures. Due to spatial hole burning, the transverse mode patterns of wide stripe, gain guided lasers do not remain stable if the current is increased much above threshold. The output beams of gain guided lasers can also have a large amount of astigmatism. Recently, there has been a great amount of work done, both experimentally and theoretically ${ }^{1-3}$ on the influence of the stripe width on the properties of gain guided lasers. The problem of spatial hole burning has been found to be greatly reduced in narrow stripe lasers, such as the V-groove laser. ${ }^{4}$ Narrow stripe lasers having very large astigmatism factors have been reported. These lasers also operate in many longitudinal modes which have been explained by the high spontaneous emission factors expected in lasers with large astigmatism. ${ }^{5,6}$ In this letter, we report on gain guided lasers in which the current is confined to flow between two narrow stripes located above and below the active layer. This laser structure is a modification of the double current confinement (DCC) configuration developed by Tsang and Logan. ${ }^{7}$ In the original DCC structure it was reported to be difficult to obtain injecting stripes with widths less than $10 \mu$. The structure which we are reporting on is capable of restricting carrier injection to an extremely narrow width of the active layer. Injecting stripe widths of $2 \mu$ were routinely obtained and injecting stripe widths as narrow as $0.5 \mu$ have been obtained. Our main emphasis in this letter will be simply describing the structure and the properties of these lasers. However, we believe the narrow stripe DCC configuration has potential applications in the fabrication of low threshold laser structures and arrays of optically coupled lasers.

The narrow stripe DCC laser structure is shown in Fig. 1. Current confinement is provided by an oxide stripe above the active layer and by $n$-type blocking layers below the active layer. The fabrication of the narrow stripe DCC structure requires only one liquid phase epitaxial (LPE) growth and is based on the fact that LPE crystal proceeds in such a manner as to flatten out any features such as mesas or chan- nels that are present in the substrate prior to the growth. In the case of GaAs substrates with etched mesas, in the first stages of the LPE growth, the tops of the mesas can be being melted back at the same time that AlGaAs layers are being grown on the sides of the mesas. Figure 2(a) shows a scanning electron microscope (SEM) photograph of the mesas that were etched in the substrate before the growth. Figure 2(b) shows the growth that results when the substrates were placed under a solution of gallium, saturated with $\mathrm{GaAs}$, for $30 \mathrm{~s}$. As can be seen, in these $30 \mathrm{~s}$, the mesa was melted back to approximately the position of the waist of the mesa, while at the same time layers were being grown on the sides of the mesa. In the narrow stripe DCC laser structure this effect is used to grow a blocking layer, which restricts the current to flow through a narrow mesa etched in the substrate prior to the growth. The layers grown for the narrow stripe DCC lasers are shown in Fig. 3. If the blocking layer is grown for a sufficiently short time it will not grow at all above the mesa. In practice, we found that when we used $n$-type substrates, the $I-V$ characteristics of the devices sometimes indicated the presence of a thin blocking layer above the mesa. However, if $\mathrm{Zn}$-doped substrates are used this problem is avoided due to the diffusion of $\mathrm{Zn}$ through any thin blocking layers that may form above the mesas. LPE growth was carried out at an initial temperature of $800^{\circ} \mathrm{C}$ and at a cooling rate of $0.4^{\circ} \mathrm{C} / \mathrm{min}$. With these growth conditions the DCC configuration could be obtained for blocking layer growth times between $45 \mathrm{~s}$ and $3 \mathrm{~min}$. For growth times less than $45 \mathrm{~s}$ the blocking layer is too thin and for growth times greater than 3

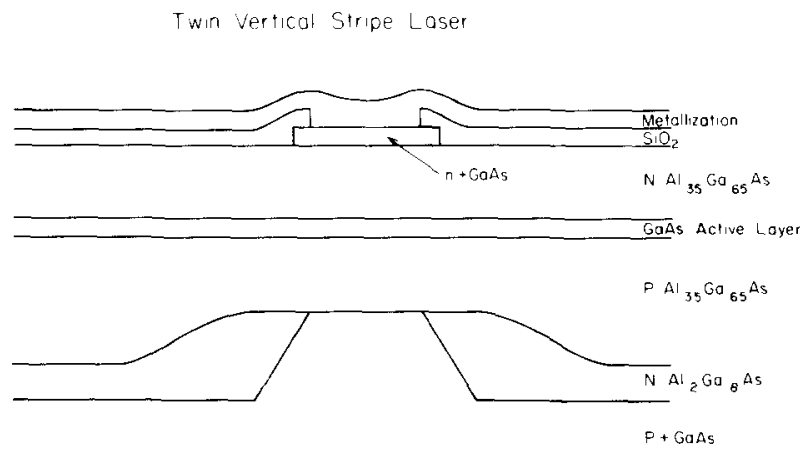

FIG. 1. Schematic diagram of the narrow stripe DCC laser structure. 


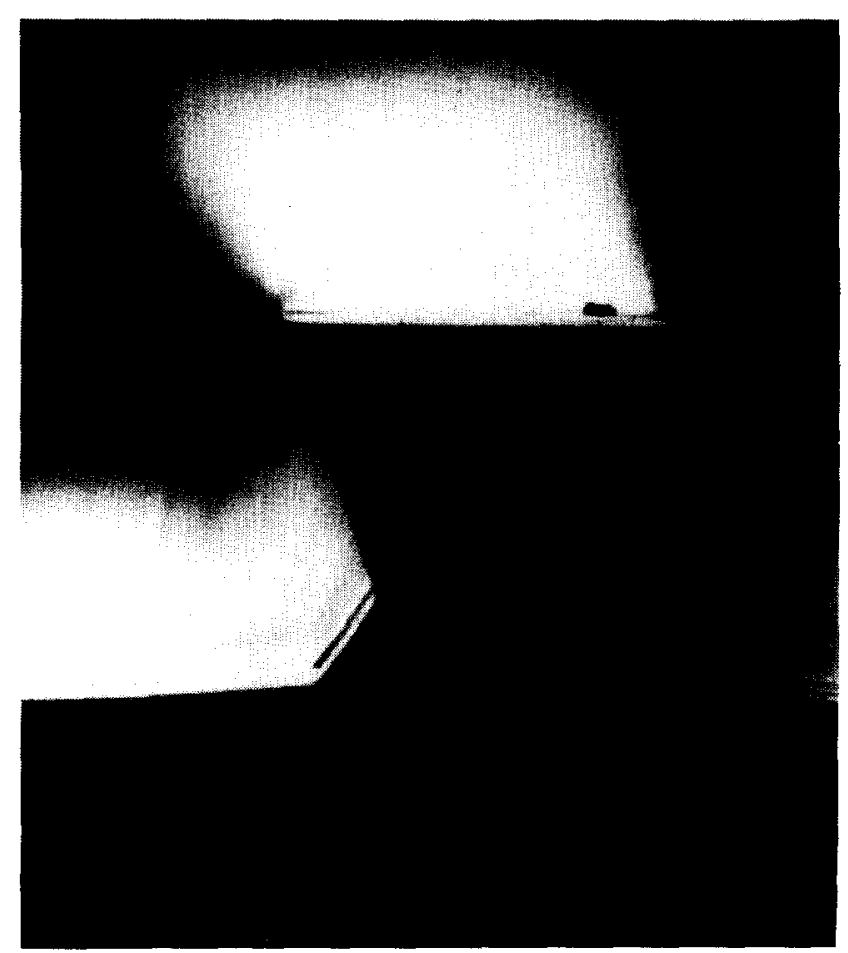

(a)

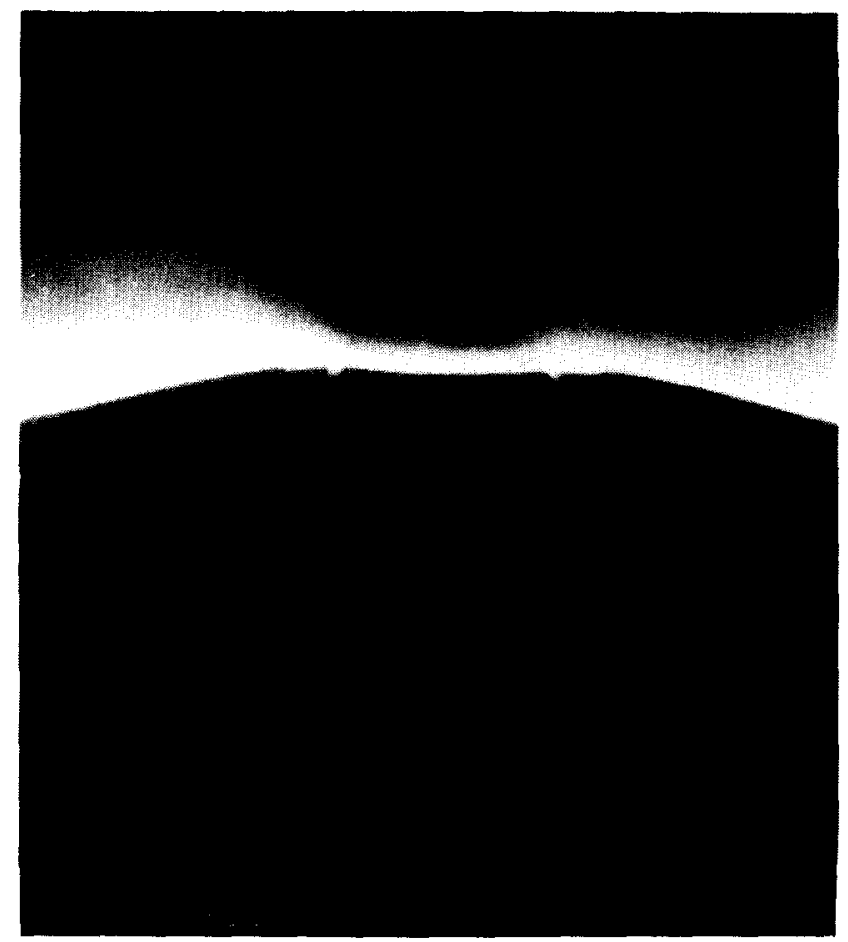

(b)

FIG. 2. (a) SEM photograph of a substrate prior to growth. (b) Growth resulting from $30 \mathrm{~s}$ under a saturated solution.

min growth on top of the mesas results. For growth times within this range the meltback and growth process was found to be very reproducible, provided the etching of the mesa in the substrate prior to the growth was carefully controlled. One significant feature of this structure is that the lower injecting stripe can be very narrow. Injecting stripes as narrow as $0.5 \mu \mathrm{m}$ have been fabricated using this technique.

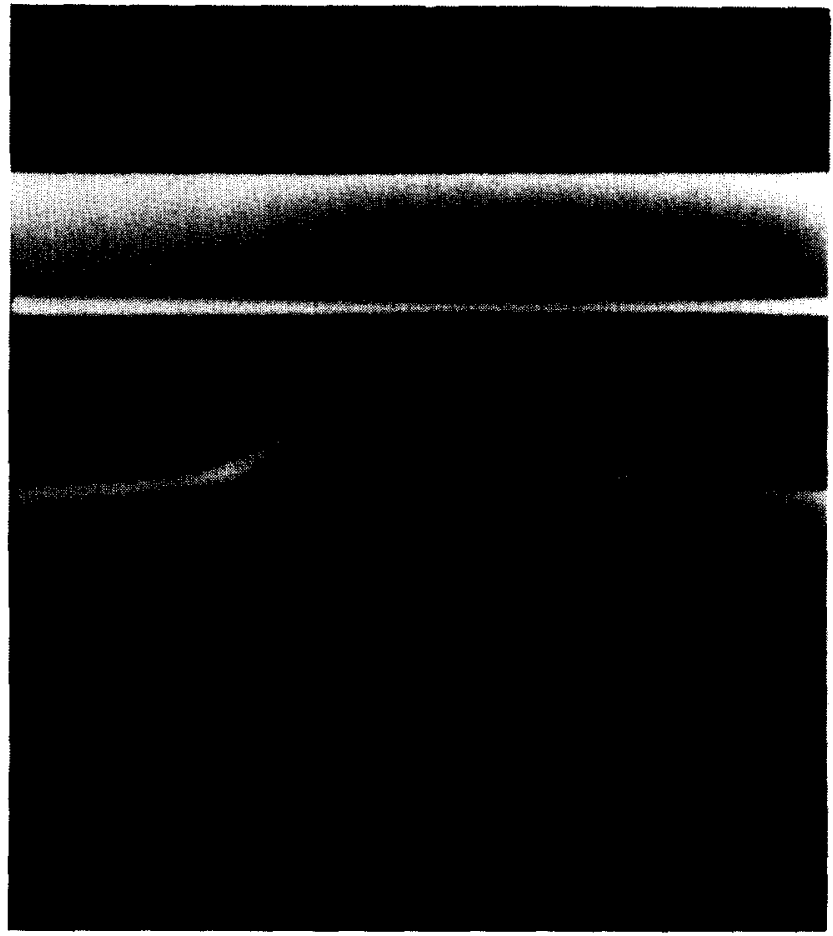

FIG. 3. Layers grown for the narrow stripe DCC laser structure.

Narrow stripe DCC lasers were found to have several interesting properties. The results to be presented were obtained for a laser with the features listed below. The width of the top GaAs contact layer and the opening in the $\mathrm{SiO}_{2}$ were $3 \mu \mathrm{m}$, the width of the lower injecting stripe was $2 \mu \mathrm{m}$, the lower and upper cladding layers were $1 \mu \mathrm{m}$ thick and doped with $\mathrm{Ge}$ and $\mathrm{Sn}$ respectively to approximately $5 \times 10^{16}$ $\mathrm{cm}^{-3}$. As is the case with other narrow stripe lasers the threshold currents of these lasers are relatively high. Threshold currents varied widely ranging from 70-150 mA for 250$\mu \mathrm{m}$-long devices. Differential quantum efficiencies were typically 15-20\% per facet. The characteristics temperature $T_{0}$ for these lasers was a relatively high $170{ }^{\circ} \mathrm{K}$. One of the most interesting properties of these lasers are the far-field patterns which are characteristic of a leaky guide. Figure 4 shows the far-field pattern of one of these lasers in the direction parallel to the junction. The lasers with threshold currents at the high end of the $70-150-\mathrm{mA}$ range were found to have far-field patterns with the most pronounced antiguiding characteristics. The variation in the threshold currents is believed to be due variations in the width of the injecting stripe with the high threshold lasers corresponding to the devices with the most narrow injection. Both the far-field patterns and the relatively high threshold currents can be understood by the fact that the real index of refraction is suppressed in the gain region by the high carrier density present there. The resulting strong real index antiguiding causes both the leaky mode far-field patterns and the high threshold currents. Far-field patterns exhibiting leaky mode characteristics have been reported previously in narrow stripe lasers; however, the effect was much more pronounced in these narrow stripe DCC lasers. This is an indication of the very narrow injection that can be achieved in 


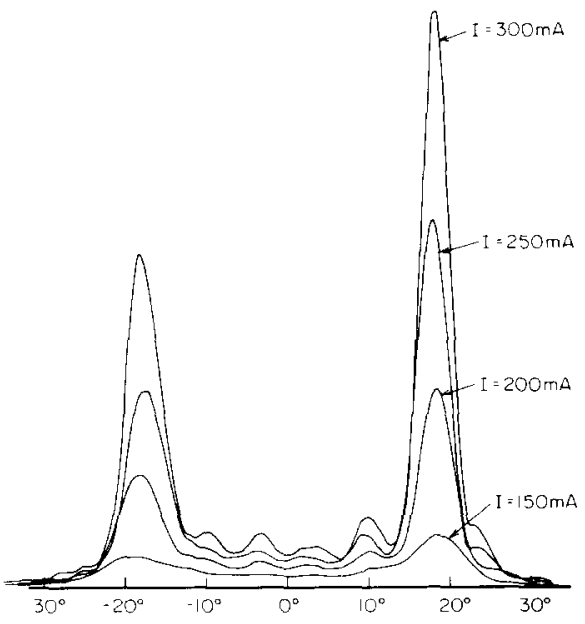

FIG. 4. Lateral far-field pattern of a narrow stripe DCC laser.

these lasers. A second property of narrow stripe DCC lasers was that they operated in a large number of longitudinal modes. The spectrum of a narrow stripe DCC laser under pulsed operation is shown in Fig. 5. This is consistent with previous findings that the number of longitudinal modes of narrow stripe lasers increases as the stripe width is decreased. $^{6}$

Although the primary purpose of this letter is to report on the properties of DCC lasers with very narrow injection, we believe that there are several advantages of the technique of injecting current through a narrow stripe in the substrate that may result in useful application for this technique. First, this technique enables the fabrication of narrow stripe lasers with broad area contacts, thus reducing contact resistance. In addition to lasers with a narrow top contact described above, we have fabricated lasers with top stripe widths of 15 $\mu \mathrm{m}$. These lasers do not have a pronounced antiguiding characteristics as the narrow stripe DCC lasers. They also have lower threshold currents than the DCC lasers, with some devices having threshold currents as low as $50 \mathrm{~mA}$. By incorporating some real index waveguiding mechanism, to provide good optical confinement, it may be possible to fabricate lasers with still lower thresholds using this technique of restricted injection through the substrate. In addition, we believe that the leaky guide nature of the narrow stripe DCC

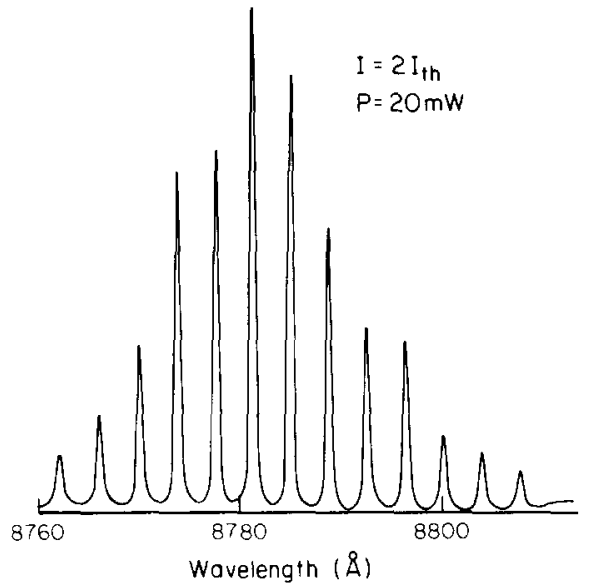

FIG. 5. Spectrum of a narrow stripe DCC laser.

structure combined with the simplicity of its fabrication makes the narrow stripe DCC structure an excellent choice for the fabrication of arrays of optically coupled lasers.

In conclusion, double current confinement lasers which feature very narrow carrier injection have been fabricated. These lasers exhibit strong antiguiding resulting in far fields characteristic of leaky waveguides. They have also been observed to operate in a large number of longitudinal modes. Finally, the technique of current injection through a narrow stripe in the substrate appears to be well suited to several applications such as laser arrays, and also to low threshold laser structures if combined with a real index waveguiding mechanism.

This research was supported by the National Science Foundation and the Office of Naval Research.

\footnotetext{
'P. M. Asbeck, D. Cammack, J. Daniele, and V. Klebanoff, IEEE J. Quantum Electron. QE-15, 727 (1979).

${ }^{2}$ R. Lang, IEEE J. Quantum Electron. QE-15, 718 (1979).

${ }^{3}$ W. Streifer, R. Burnham, and D. Scifres, IEEE J. Quantum Electron. QE18, $856(1982)$.

${ }^{4}$ C. Wolk, H. Gottsmann, P. Marschall; K. Peterman, W. Pfister, and H. Vollmer, IEEE J. Quantum Electron. QE-17, 756 (1981).

${ }^{5}$ K. Petermann, IEEE J. Quantum. Electron. QE-15, 566 (1979).

${ }^{6} \mathrm{~W}$. Streiffer, D. Scifres, and R. Burnham, Appl. Phys. Lett. 40, 305 (1982).

${ }^{7}$ W. T. Tsang and R. Logan, J. Appl. Phys. 49, 2629 (1978).
} 